\title{
THE SIGNATURE OF THE CHERN COEFFICIENTS OF LOCAL RINGS
}

\author{
Laura Ghezzi, Jooyoun Hong, and Wolmer V. Vasconcelos
}

\begin{abstract}
This paper considers the following conjecture: If $R$ is an unmixed, equidimensional local ring that is a homomorphic image of a Cohen-Macaulay local ring, then for any ideal $J$ generated by a system of parameters, the Chern coefficient $e_{1}(J)<0$ is equivalent to $R$ being non Cohen-Macaulay. The conjecture is established if $R$ is a homomorphic image of a Gorenstein ring, and for all universally catenary integral domains containing fields. Criteria for the detection of Cohen-Macaulayness in equi-generated graded modules are derived.
\end{abstract}

\section{Introduction}

Let $(R, \mathfrak{m})$ be a Noetherian local ring of dimension $d>0$, and let $I$ be an $\mathfrak{m}$ primary ideal. We will consider the set of multiplicative, decreasing filtrations of $R$ ideals, $\mathbf{B}=\left\{I_{n}, I_{0}=R, I_{n+1}=I I_{n}, n \gg 0\right\}$, integral over the $I$-adic filtration. They are conveniently coded in the corresponding Rees algebra and its associated graded ring

$$
\mathcal{R}(\mathbf{B})=\sum_{n \geq 0} I_{n} t^{n}, \quad \operatorname{gr}_{\mathbf{B}}(R)=\sum_{n \geq 0} I_{n} / I_{n+1} .
$$

One of our goals is to study cohomological properties of these filtrations. For that we will focus on the role of the Hilbert polynomial of the Hilbert-Samuel function $\lambda\left(R / I_{n+1}\right)$,

$$
H_{\mathbf{B}}^{1}(n)=P_{\mathbf{B}}^{1}(n) \equiv \sum_{i=0}^{d}(-1)^{i} e_{i}(\mathbf{B})\left(\begin{array}{c}
n+d-i \\
d-i
\end{array}\right),
$$

particularly of the multiplicity, $e_{0}(\mathbf{B})$, and the Chern coefficient, $e_{1}(\mathbf{B})$. For CohenMacaulay rings, many penetrating relationships between these coefficients have been proved, beginning with Northcott's [7]. More recently, similar questions have been examined in general Noetherian local rings and among those pertinent to our concerns are [3], [10] and [12].

Here we extend several of the results of [12] on the meaning of the sign of $e_{1}(\mathbf{B})$, particularly in the case of $I$-adic filtrations. Our main results are centered around the following question:

Let $J$ be an ideal generated by a system of parameters. Under which conditions is $e_{1}(J)<0$ equivalent to $R$ being not Cohen-Macaulay? We conjecture that this is so

Received by the editors July 14, 2008.

AMS 2000 Mathematics Subject Classification. Primary 13A30; Secondary 13B22, 13H10, 13H15.

The first author is partially supported by a grant from the City University of New York PSCCUNY Research Award Program-39. The third author is partially supported by the NSF. 
whenever $R$ is an unmixed, equidimensional local ring that is a homomorphic image of a Cohen-Macaulay local ring.

There are reasons for the interest in these numbers. To make the discussion more direct, we assume that the residue field of $R$ is infinite.

(1) Clarifying the role of the sign of $e_{1}(J)$ in the Cohen-Macaulay property of $R$ could be used as a scale to classify non-CM rings.

(2) In the study of the normalization $\mathbf{B}$ of $R[J t]$, the expression (see [8], [12])

$$
e_{1}(\mathbf{B})-e_{1}(J)
$$

bounds the length of certain computations in the construction of normalizations. It highlights the need to look for upper bounds of $e_{1}(\mathbf{B})$ and for lower bounds of $e_{1}(J)$.

(3) The value of $e_{1}(J)$ occurs as a correction term in the extensions of several well-known formulas in the theory of the Hilbert polynomials. We highlight two of them. A classical result of Northcott ([7]) asserts that if $R$ is Cohen-Macaulay, then

$$
e_{1}(I) \geq e_{0}(I)-\lambda(R / I) \text {. }
$$

For arbitrary Noetherian rings, if $J$ is a minimal reduction of $I$, Goto and Nishida ([3, Theorem 3.1])proved that

$$
e_{1}(I)-e_{1}(J) \geq e_{0}(I)-\lambda(R / I)
$$

which gives

$$
e_{1}(\mathbf{B})-e_{1}(J) \geq e_{0}(I)-\lambda(R / \bar{I})
$$

since $e_{1}(\mathbf{B}) \geq e_{1}(I)$ for all such ideals $I$. It is a formula which is relevant to a conjecture of [12], on whether $e_{1}(\mathbf{B}) \geq 0$.

A different kind of relationship given by Huckaba and Marley ([4, Theorem 4.7]) for Cohen-Macaulay rings,

$$
e_{1}(\mathbf{B}) \leq \sum_{n \geq 1} \lambda\left(I_{n} / J I_{n-1}\right)
$$

is extended by Rossi and Valla ([10, Theorem 2.11]) to general filtrations to an expression that replaces $e_{1}(\mathbf{B})$ in the inequality above by $e_{1}(\mathbf{B})-e_{1}(J)$.

In our main result (Theorem 3.3) we show that the above Conjecture holds if $R$ is a homomorphic image of a Gorenstein ring, or milder extensions that allow an embedding of $R$ into a (small) Cohen-Macaulay module over a possibly larger ring. The proof is a variation of an argument in [12], but turned more abstract. Another result establishes the Conjecture for universally catenary local domains containing a field (Theorem 4.4).

The same question can be asked about filtrations of modules regarding the negativity of the coefficient $e_{1}$ of the corresponding associated graded module. In case $R$ is a polynomial ring over a field and $M$ is a graded, torsion-free $R$-module, this extension has a surprising application to the Cohen-Macaulayness of $M$. In the special case of modules generated in the same degree, the Hilbert coefficient $e_{1}(M)$, which may be different from $e_{1}\left(\operatorname{gr}_{\mathbf{x}}(M)\right)$, can alone decide whether $M$ is Cohen-Macaulay or not (Corollary 5.4) .

We thank Rodney Sharp and Santiago Zarzuela for sharing with us their expertises on balanced big Cohen-Macaulay modules. We are also grateful to Shiro Goto for 
sharing with us a sketch of his proof of a more extended version of Theorem 3.3. The Yokohama Conference on Commutative Algebra, on March 2008, was the setting where some of the questions arose.

\section{Preliminaries}

We will assemble quickly here some facts about associated graded modules and their Hilbert functions. As a general reference for unexplained terminology and basic results, we shall use [1].

Let $(R, \mathfrak{m})$ be a Noetherian local ring, $I$ an $\mathfrak{m}$-primary ideal, and $M$ a nonzero finite $R$-module of dimension $d$. The associated graded ring $\operatorname{gr}_{I}(R)=\bigoplus_{i=0}^{\infty} I^{i} / I^{i+1}$ is a standard graded ring with $\left[\operatorname{gr}_{I}(R)\right]_{0}=R / I$ Artinian. The associated graded module $\operatorname{gr}_{I}(M)=\bigoplus_{i=0}^{\infty} I^{i} M / I^{i+1} M$ of $I$ with respect to $M$ is a finitely generated graded $\operatorname{gr}_{I}(R)$-module. The Hilbert-Samuel function $\chi_{M}^{I}(n)$ of $M$ with respect to $I$ is

$$
\chi_{M}^{I}(n)=\lambda\left(M / I^{n+1} M\right)=\sum_{i=0}^{n} \lambda\left(I^{i} M / I^{i+1} M\right) .
$$

For sufficiently large $n$, the Hilbert-Samuel function $\chi_{M}^{I}(n)$ is of polynomial type :

$$
\chi_{M}^{I}(n)=\sum_{i=0}^{d}(-1)^{i} e_{i}(I, M)\left(\begin{array}{c}
n+d-i \\
d-i
\end{array}\right) .
$$

For an $R$-module $M$ of finite length, we denote the length of $M$ by $\lambda(M)$.

Lemma 2.1. Let $(R, \mathfrak{m})$ be a Noetherian local ring and let $I$ be an $\mathfrak{m}$-primary ideal. Let $0 \rightarrow T \rightarrow M \rightarrow N \rightarrow 0$ be an exact sequence of finitely generated $R$-modules. Assume that $M$ has dimension $d \geq 2$ and that $T$ has finite length. Then $e_{1}(I, M)=$ $e_{1}(I, N)$.

Proof. From the following commutative diagram with exact rows

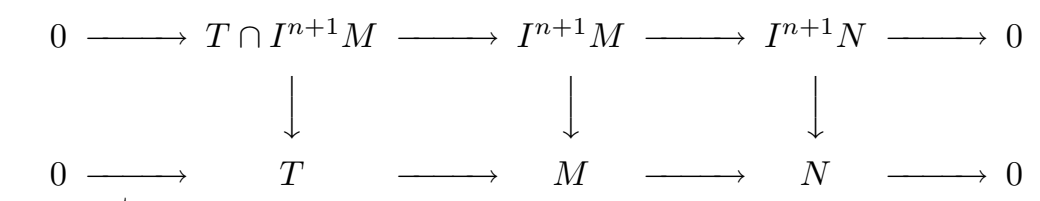

we get an exact sequence

$$
0 \longrightarrow T /\left(T \cap I^{n+1} M\right) \longrightarrow M / I^{n+1} M \longrightarrow N / I^{n+1} N \longrightarrow 0 .
$$

By the Artin-Rees Lemma, $T \cap I^{n+1} M=0$ for all sufficiently large $n$. Hence we get for all $n \gg 0$

$$
\lambda(T)-\lambda\left(M / I^{n+1} M\right)+\lambda\left(N / I^{n+1} N\right)=0 .
$$

Let $d^{\prime}$ be the dimension of $N$. There are Hilbert polynomials such that

$$
\lambda\left(M / I^{n+1} M\right)=\sum_{i=0}^{d}(-1)^{i} e_{i}(I, M)\left(\begin{array}{c}
d+n-i \\
d-i
\end{array}\right)
$$


and

$$
\lambda\left(N / I^{n+1} N\right)=\sum_{i=0}^{d^{\prime}}(-1)^{i} e_{i}(I, N)\left(\begin{array}{c}
d^{\prime}+n-i \\
d^{\prime}-i
\end{array}\right)
$$

for all sufficiently large $n$. Therefore $d=d^{\prime}$,

$e_{i}(I, M)=e_{i}(I, N) \quad$ for all $i=0, \ldots, d-1$ and $e_{d}(I, N)=e_{d}(I, M)+(-1)^{d+1} \lambda(T)$.

In particular, since $d \geq 2$, we have $e_{1}(I, M)=e_{1}(I, N)$.

Let $R$ be a ring, $I$ an $R$-ideal and $M$ an $R$-module. An element $h \in I$ is called a superficial element of $I$ with respect to $M$ if there exists a positive integer $c$ such that

$$
\left(I^{n+1} M:_{M} h\right) \cap I^{c} M=I^{n} M
$$

for all $n \geq c$. A detailed discussion on superficial elements can be found in several sources, but we especially benefited from the treatment in [9, Theorem 1.5] of the construction of superficial elements. It depends simply on showing that certain finitely generated modules cannot be written as a union of a finite set of proper submodules. The existence of such elements is guaranteed if the residue field of $R$ is infinite. Its usefulness for our purposes is expressed in the following result.

Proposition 2.2. $([6,(22.6)])$ Let $(R, \mathfrak{m})$ be a Noetherian local ring, I an $\mathfrak{m}$-primary ideal, and $M$ a nonzero finitely generated $R$-module of dimension $d$. Let $h$ be a superficial element of $I$ with respect to $M$. Then the Hilbert coefficients of $M$ and $M / h M$ satisfy

$$
e_{i}(I, M)= \begin{cases}e_{i}(I /(h), M / h M) & \text { for } \quad i<d-1 . \\ e_{d-1}(I /(h), M / h M)+(-1)^{d-1} \lambda\left(0:_{M} h\right) . & \text { for } \quad i=d-1 .\end{cases}
$$

\section{Cohen-Macaulayness versus the vanishing of the Euler number}

In this section we develop an abstract approach to the relationship between the signature of $e_{1}$ and the Cohen-Macaulayness of a local ring (see [12, Theorem 3.1]). We also give a more general but still self-contained proof of the main result of [12] that avoids the use of big Cohen-Macaulay modules.

Lemma 3.1. Let $(S, \mathfrak{n})$ be a Cohen-Macaulay local ring of dimension d with infinite residue field and let $R=S / \mathfrak{p}$, where $\mathfrak{p}$ is a minimal prime ideal of $S$. Let $x_{1}, \ldots, x_{d}$ be a system of parameters of $R$. Then there exists a system of parameters $a_{1}, \ldots, a_{d}$ of $S$ such that $x_{i}=a_{i}+\mathfrak{p}$ for each $i$.

Proof. Let $\mathfrak{m}$ denote the maximal ideal of $R$ and let $\mathfrak{p}=\left(c_{1}, \ldots, c_{s}\right)$. Let $x_{1}=b_{1}+\mathfrak{p}$ for some $b_{1} \in S$ and let $\mathfrak{p}_{1}, \ldots, \mathfrak{p}_{n}$ be the minimal primes of $S$ different from $\mathfrak{p}$. We claim that there exists $\lambda \in S \backslash \mathfrak{n}$ such that $b_{1}+\lambda c_{1}+\cdots+\lambda^{s} c_{s} \notin \mathfrak{p}_{i}$ for all $i=1, \ldots, n$. Suppose not: since $S / \mathfrak{n}$ is infinite, there exist $\lambda_{1}, \ldots, \lambda_{s+1} \in S \backslash \mathfrak{n}$ such that $\lambda_{i}+\mathfrak{n} \neq \lambda_{j}+\mathfrak{n}$ whenever $i \neq j$ and such that $b_{1}+\lambda_{i} c_{1}+\cdots+\lambda_{i}^{s} c_{s} \in \mathfrak{p}_{k}$ for some fixed $k$. Let $A$ be the Vandermonde matrix determined by $\lambda_{i}, 1 \leq i \leq s+1$. We have

$$
A\left[\begin{array}{c}
b_{1} \\
c_{1} \\
\vdots \\
c_{s}
\end{array}\right]=\left[\begin{array}{ccccc}
1 & \lambda_{1} & \lambda_{1}^{2} & \cdots & \lambda_{1}^{s} \\
1 & \lambda_{2} & \lambda_{2}^{2} & \cdots & \lambda_{2}^{s} \\
\vdots & \vdots & \vdots & \ddots & \vdots \\
1 & \lambda_{s+1} & \lambda_{s+1}^{2} & \cdots & \lambda_{s+1}^{s}
\end{array}\right]\left[\begin{array}{c}
b_{1} \\
c_{1} \\
\vdots \\
c_{s}
\end{array}\right]=\left[\begin{array}{c}
g_{1} \\
g_{2} \\
\vdots \\
g_{s+1}
\end{array}\right],
$$


where $g_{1}, \ldots, g_{s+1} \in \mathfrak{p}_{k}$. Since $A$ is invertible, $c_{1}, \ldots, c_{s} \in \mathfrak{p}_{k}$ so that $\mathfrak{p} \subseteq \mathfrak{p}_{k}$, a contradiction. Let $a_{1}=b_{1}+\lambda c_{1}+\cdots+\lambda^{s} c_{s}$, with $\lambda \in S \backslash \mathfrak{n}$, be such that $a_{1} \notin \mathfrak{p}_{i}$ for all $i=1, \ldots, n$. Hence $a_{1}$ is not contained in any minimal prime ideal of $S$ and $a_{1}+\mathfrak{p}=x_{1}$.

Let $\mathfrak{q}_{1}, \ldots, \mathfrak{q}_{m}$ be the minimal primes of $a_{1} S$ that do not contain $\mathfrak{p}$. Let $x_{2}=b_{2}+\mathfrak{p}$ for some $b_{2} \in S$. Then similarly as shown above, there exists $\tau \in S \backslash \mathfrak{n}$ such that $b_{2}+\tau c_{1}+\cdots+\tau^{s} c_{s} \notin \mathfrak{q}_{i}$ for all $i$. Let $a_{2}=b_{2}+\tau c_{1}+\cdots+\tau^{s} c_{s}$. Then $a_{2}$ is not contained in any minimal prime ideal of $a_{1} S$ and $a_{2}+\mathfrak{p}=x_{2}$. Now inductively we show that there exists a system of parameters $a_{1}, \ldots, a_{d}$ of $S$ such that $a_{i}+\mathfrak{p}=x_{i}$ for all $i$.

Lemma 3.2. Let $(S, \mathfrak{n})$ be a Cohen-Macaulay complete local ring of dimension $d \geq 2$ and let $M$ be a finitely generated $S$-module of dimension d satisfying Serre's condition $\left(S_{1}\right)$. Then $H_{\mathfrak{n}}^{1}(M)$ is a finitely generated $S$-module.

Proof. Let $k$ denote the residue field of $S$ and $\omega$ the canonical module of $S$. Since $M$ satisfies Serre's condition $\left(S_{1}\right), \operatorname{Ext}_{S}^{d-1}(M, \omega)$ is zero at each localization at $\mathfrak{p}$ such that $\mathfrak{p} \neq \mathfrak{n}$. Thus it is a module of finite length. By Grothendieck duality $([1,3.5 .8])$, we have

$$
H_{\mathfrak{n}}^{1}(M) \simeq \operatorname{Hom}_{S}\left(\operatorname{Ext}_{S}^{d-1}(M, \omega), E(k)\right) .
$$

By Matlis duality $([1,3.2 .13]), H_{\mathfrak{n}}^{1}(M)$ is finitely generated.

Let $(R, \mathfrak{m})$ be a Noetherian local ring of dimension $d \geq 2$. The enabling idea is the embedding of $R$ into a Cohen-Macaulay (possibly big Cohen-Macaulay) module $E$,

$$
0 \rightarrow R \longrightarrow E \longrightarrow C \rightarrow 0 \text {. }
$$

Unfortunately, it may not be always possible to find the appropriate $R$-module $E$. Instead we will seek embed $R$ into a Cohen-Macaulay module $E$ over a ring $S$ closely related to $R$ for the purpose of computing associated graded rings of adic-filtrations. The following is our main result.

Theorem 3.3. Let $(R, \mathfrak{m})$ be a Noetherian local domain of dimension $d \geq 2$, which is a homomorphic image of a Cohen-Macaulay local ring $(S, \mathfrak{n})$. If $R$ is not CohenMacaulay, then $e_{1}(J)<0$ for any $R$-ideal $J$ generated by a system of parameters.

Proof. Let $R=S / \mathfrak{p}$. We may assume that $S$ has infinite residue field. If height $(\mathfrak{p}) \geq 1$, we replace $S$ by $S / L$, where $L$ is the $S$-ideal generated by a maximal regular sequence in $\mathfrak{p}$. This means that we may assume that $\operatorname{dim} R=\operatorname{dim} S$, and that $\mathfrak{p}$ is a minimal prime of $S$. In particular, we have an exact sequence of $S$-modules

$$
0 \longrightarrow R \longrightarrow S \longrightarrow C \longrightarrow 0 \text {. }
$$

Let $J=\left(x_{1}, \ldots, x_{d}\right)$ be an $R$-ideal generated by a system of parameters. Then by Lemma 3.1, there exists a system of parameters $a_{1}, \ldots, a_{d}$ of $S$ such that $x_{i}=a_{i}+\mathfrak{p}$ for each $i$. Let $I=\left(a_{1}, \ldots, a_{d}\right) S$. Since $I R=J$, the associated graded $\operatorname{ring} \operatorname{gr}_{I}(R)$ of $I$ with respect to the $S$-module $R$ is equal to the associated graded $\operatorname{ring} \operatorname{gr}_{J}(R)$ of $J$. In particular, $e_{1}(I, R)=e_{1}(J)$. Therefore, for the purpose of constructing $e_{1}(J)$, we treat $R$ as an $S$-module and use the $I$-adic filtration. 
Now proceed as in the proof of [12, Theorem 3.1]. Let $a$ be a superficial element for $I$ with respect to the $S$-module $R$ which is not contained in any associated prime of $C$ distinct from $\mathfrak{n}$. We may assume that $a=a_{1}$. Reduction modulo $a S$ gives rise to the exact sequence

$$
0 \rightarrow T=\operatorname{Tor}_{1}^{S}(S / a S, C) \longrightarrow R / a R \longrightarrow S / a S \longrightarrow C / a C \rightarrow 0,
$$

where $T=\left(0::_{C} a\right) \subset C$. This shows that the associated primes of $T$ contain $a$. Therefore $T$ is either zero, or $T$ is a non-zero module of finite support.

Let $R^{\prime}=R / a R, I^{\prime}=I /(a)$, and denote the image of $R / a R$ in $S / a S$ by $S^{\prime}$.

Now we use induction on $d \geq 2$ to show that if $R$ is not Cohen-Macaulay, then $e_{1}(I, R)<0$. Let $d=2$. Notice that $S^{\prime}$ is a Cohen-Macaulay ring of dimension 1 . We have that depth $C=0$, and so $T \neq 0$. As in the proof of [12, Theorem 3.1], we obtain $e_{1}\left(I^{\prime}, R^{\prime}\right)=-\lambda(T)$. Hence by Proposition 2.2, $e_{1}(I, R)=-\lambda(T)<0$.

Suppose $d>2$. Consider the exact sequence of $S / a S$-modules $: 0 \rightarrow T \rightarrow R^{\prime} \rightarrow$ $S^{\prime} \rightarrow 0$. By Lemma 2.1, we have $e_{1}\left(I^{\prime}, R^{\prime}\right)=e_{1}\left(I^{\prime}, S^{\prime}\right)$ since $\operatorname{dim}(S / a S)=d-1 \geq 2$. Now it is enough to show that $S^{\prime}$ is not a Cohen-Macaulay $S / a S$-module. Then since $\operatorname{dim}\left(S^{\prime}\right)=d-1$, by induction we get $e_{1}\left(I^{\prime}, S^{\prime}\right)<0$, and we conclude using Proposition 2.2.

Suppose that $S^{\prime}$ is a Cohen-Macaulay $S / a S$-module. Let $\mathfrak{n}$ denote the maximal ideal of $S / a S$ as well and let $H_{\mathfrak{n}}^{i}(\cdot)$ denote the $i$ th local cohomology. We are going to use the argument of [5, Proposition 2.1]. From the exact sequence $0 \rightarrow T \rightarrow R^{\prime} \rightarrow S^{\prime} \rightarrow 0$, we obtain a long exact sequence:

$$
0 \rightarrow H_{\mathfrak{n}}^{0}(T) \rightarrow H_{\mathfrak{n}}^{0}\left(R^{\prime}\right) \rightarrow H_{\mathfrak{n}}^{0}\left(S^{\prime}\right) \rightarrow H_{\mathfrak{n}}^{1}(T) \rightarrow H_{\mathfrak{n}}^{1}\left(R^{\prime}\right) \rightarrow H_{\mathfrak{n}}^{1}\left(S^{\prime}\right) .
$$

Since $S^{\prime}$ is Cohen-Macaulay of dimension $d-1 \geq 2$, we have $H_{\mathfrak{n}}^{0}\left(S^{\prime}\right)=0=H_{\mathfrak{n}}^{1}\left(S^{\prime}\right)$. Since $T$ is a torsion module, we have $H_{\mathfrak{n}}^{0}(T)=T$ and $H_{\mathfrak{n}}^{1}(T)=0$. Therefore $T \simeq$ $H_{\mathfrak{n}}^{0}\left(R^{\prime}\right)$ and $H_{\mathfrak{n}}^{1}\left(R^{\prime}\right)=0$. Now from the exact sequence of $S$-modules

$$
0 \longrightarrow R \stackrel{\cdot a}{\longrightarrow} R \longrightarrow R / a R \longrightarrow 0
$$

we obtain the following exact sequence:

$$
0 \longrightarrow T \simeq H_{\mathfrak{n}}^{0}\left(R^{\prime}\right) \longrightarrow H_{\mathfrak{n}}^{1}(R) \stackrel{\cdot a}{\longrightarrow} H_{\mathfrak{n}}^{1}(R) \longrightarrow H_{\mathfrak{n}}^{1}\left(R^{\prime}\right)=0 .
$$

Therefore $H_{\mathfrak{n}}^{1}(R)=a H_{\mathfrak{n}}^{1}(R)$. Moreover, once $R$ is embedded in $S$, we may assume that $S$ is a complete local ring. By Lemma $3.2, H_{\mathfrak{n}}^{1}(R)$ is finitely generated. By Nakayama Lemma, we have that $H_{\mathfrak{n}}^{1}(R)=0$ so that $T=0$. It follows that $R / a R=R^{\prime} \simeq S^{\prime}$, where $S^{\prime}$ is Cohen-Macaulay. This means that $R$ is Cohen-Macaulay, which is a contradiction.

Remark 3.4. The proof of Theorem 3.3 can be extended from integral domains to more general local rings, $R=S / L$, where $S$ is Cohen-Macaulay and $\operatorname{dim} R=\operatorname{dim} S$, if $R$ can be embedded into a maximal Cohen-Macaulay $S$-module. Notice that in order to embed $R$ into a maximal Cohen-Macaulay $S$-module at a minimum we need to require that $R$ be unmixed and equidimensional.

There is room for the following problem: 
Problem 3.5. Let $R=S / L$ (unmixed and equidimensional as above), where $S$ is a Cohen-Macaulay local ring of $\operatorname{dimension} \operatorname{dim} R$. Characterize those $R$ that can be embedded into a maximal Cohen-Macaulay $S$-module. Note that $L$ may be assumed to be a primary ideal: If $L=\cap Q_{i}$ is a primary decomposition, we have the embedding

$$
S / L \hookrightarrow S / Q_{1} \oplus \cdots \oplus S / Q_{n} .
$$

We make an elementary observation of what it takes to embed $R$ into a free $S$ module (see [11, Theorem A.1]).

Proposition 3.6. Let $S$ be a Noetherian ring and $L$ an ideal of codimension zero without embedded components. If $R=S / L$, there is an embedding $R \rightarrow F$ into a free $S$-module $F$ if and only if $L=0:(0: L)$. In particular, this condition always holds if the total ring of fractions of $S$ is a Gorenstein ring.

Proof. Let $\left\{a_{1}, \ldots, a_{n}\right\}$ be a generating set of $0: L$, and consider the mapping $\varphi$ : $S \rightarrow S^{n}, \varphi(1)=\left(a_{1}, \ldots, a_{n}\right)$; its kernel is isomorphic to $0:(0: L)$. This shows that the equality $L=0:(0: L)$ is required for the asserted embedding.

Conversely, given an embedding $\varphi: S / L \rightarrow S^{n}$, let $\left(a_{1}, \ldots, a_{n}\right) \in S^{n}$ be the image of a generator of $S / L$. The ideal $\mathfrak{a}$ these entries generate is annihilated by $L$, and so $\mathfrak{a} \subset 0: L$. Since $0: \mathfrak{a}=L$, we have $0:(0: L) \subset 0: \mathfrak{a}=L$.

If the total ring of fractions of $S$ is Gorenstein, to prove that $0:(0: L) \subset L$, it suffices to localize at the associated primes of $L$, all of which have codimension zero and a localization which is Gorenstein. But the double annihilator property is characteristic of such rings.

Corollary 3.7. Let $(R, \mathfrak{m})$ be an unmixed and equidimensional Noetherian local ring of dimension $d \geq 2$, which is a homomorphic image of a Gorenstein local ring. If $R$ is not Cohen-Macaulay, then $e_{1}(J)<0$ for any $R$-ideal $J$ generated by a system of parameters.

Proof. Let $R=S / L$. If height $(L) \geq 1$, we replace $S$ by $S / L^{\prime}$, where $L^{\prime}$ is the $S$-ideal generated by a maximal regular sequence in $L$. So we may assume that $\operatorname{dim} R=\operatorname{dim} S$, and the conclusion follows by Proposition 3.6 and Remark 3.4.

We give now a family of examples based on a method of [3].

Example 3.8. Let $(S, \mathfrak{m})$ be a regular local ring of dimension four, with an infinite residue field. Let $P_{1}, \ldots, P_{r}$ be a family of codimension two Cohen-Macaulay ideals such that for $i \neq j, P_{i}+P_{j}$ is an $\mathfrak{m}$-primary ideal. Define $R=S / \cap_{i} P_{i}$.

Consider the exact sequence of $S$-modules

$$
0 \rightarrow R \longrightarrow \bigoplus_{i} S / P_{i} \longrightarrow L \rightarrow 0 .
$$

Note that $L$ is a module of finite support; it may be identified with $H_{\mathfrak{m}}^{1}(R)$. Let $J=(a, b)$ be an ideal of $R$ forming a system of parameters, contained in the annihilator of $L{ }^{1}$ We can assume that $a, b \in S$ form a regular sequence in each $S / P_{i}$. We are going to determine $e_{1}(J)$.

\footnotetext{
${ }^{1}$ We thank Jugal Verma for this observation.
} 
For each integer $n$, tensoring by $S /(a, b)^{n}$ we get the exact sequence

$$
0 \rightarrow \operatorname{Tor}_{1}^{S}\left(L, S /(a, b)^{n}\right) \rightarrow R /(a, b)^{n} \rightarrow \bigoplus_{i} S /\left(P_{i},(a, b)^{n}\right) \rightarrow L \otimes_{S} S /(a, b)^{n} \rightarrow 0 .
$$

For $n \gg 0,(a, b)^{n} L=0$, so we have that $L \otimes_{S} S /(a, b)^{n}=L$ and $\operatorname{Tor}_{1}^{S}\left(L, S /(a, b)^{n}\right)=$ $L^{n+1}$, from the Burch-Hilbert $(n+1) \times n$ resolution of the ideal $(a, b)^{n}$. Since the $R_{i}=S / P_{i}$ are Cohen-Macaulay, we obtain the following Hilbert-Samuel polynomial:

$e_{0}(J)\left(\begin{array}{c}n+2 \\ 2\end{array}\right)-e_{1}(J)\left(\begin{array}{c}n+1 \\ 1\end{array}\right)+e_{2}(J)=\left(\sum_{i=1}^{r} e_{0}\left(J R_{i}\right)\right)\left(\begin{array}{c}n+2 \\ 2\end{array}\right)+(n+2) \lambda(L)-\lambda(L)$.

It gives

$$
\begin{aligned}
& e_{0}(J R)=\sum_{i=1}^{r} e_{0}\left(J R_{i}\right) \\
& e_{1}(J R)=-\lambda(L) \\
& e_{2}(J R)=0
\end{aligned}
$$

\section{Embedding into balanced big Cohen-Macaulay modules}

Let $(R, \mathfrak{m})$ be a Noetherian local domain. If $R$ has a big Cohen-Macaulay module $E$, any nonzero element of $E$ allows for an embedding $R \hookrightarrow E$. In fact, one may assume that $E$ is a balanced big Cohen-Macaulay module (see [1, Section 8.5] for a discussion). According to the results of Hochster, if $R$ contains a field, then there is a balanced big Cohen-Macaulay $R$-module $E([1,8.4 .2])$.

To use the argument in $[12$, Theorem 3.2], in the exact sequence

$$
0 \rightarrow R \longrightarrow E \longrightarrow C \rightarrow 0
$$

we should, given any parameter ideal $J$ of $R$, pick an element superficial for the purpose of building $\operatorname{gr}_{J}(R)$ (if $\operatorname{dim} R>2$ ) and not contained in any associated prime of $C$ different from $\mathfrak{m}$. This is possible if the cardinality of the residue field is larger than the cardinality of $\operatorname{Ass}(C)$.

Theorem 4.1. Let $(R, \mathfrak{m})$ be a Noetherian local integral domain that is not CohenMacaulay and let $E$ be a balanced big Cohen-Macaulay module. If the residue field of $R$ has cardinality larger than the cardinality of a generating set for $E$, then $e_{1}(J)<0$ for any parameter ideal $J$.

Let $\mathbf{X}$ be a set of indeterminates of larger cardinality than $\operatorname{Ass}(C)$, and consider $R(\mathbf{X})=R[\mathbf{X}]_{\mathfrak{m} R[\mathbf{X}]}$. This is a Noetherian ring $([2])$, and we are going to argue that if $E$ is a balanced big Cohen-Macaulay $R$-module, then $R(\mathbf{X}) \otimes_{R} E$ is a balanced big Cohen-Macaulay module over $R(\mathbf{X})$. S. Zarzuela has kindly pointed out to us the following result:

Theorem 4.2 ([13, Theorem 2.3]). Let $A \rightarrow B$ be a flat morphism of local rings $(A, \mathfrak{m}),(B, \mathfrak{n})$ and $M$ a balanced big Cohen-Macaulay A-module. Then, $M \otimes_{A} B$ is a balanced big Cohen-Macaulay B-module if and only if the following two conditions hold:

(i) $\mathfrak{n}\left(M \otimes_{A} B\right) \neq M \otimes_{A} B$ and 
(ii) For any prime ideal $\mathfrak{q} \in \operatorname{supp}_{B}\left(M \otimes_{A} B\right)$, (1) height $(\mathfrak{q} / \mathfrak{p} B)=\operatorname{depth}\left(C_{\overline{\mathfrak{q}}}\right)$ and (2) $\operatorname{height}(\mathfrak{q})+\operatorname{dim}(B / \mathfrak{q})=\operatorname{dim}(B)$.

Here, we denote by $\operatorname{supp}_{A}(M)$ (small support) the set of prime ideals in $A$ with at least one non-zero Bass number in the A-minimal injective resolution of $M, \mathfrak{p}=\mathfrak{q} \cap A$, $C=B / \mathfrak{p} B$ and $\overline{\mathfrak{q}}=\mathfrak{q} C$.

Moreover, if $\mathfrak{q} \in \operatorname{supp}_{B}\left(M \otimes_{A} B\right)$ then $\mathfrak{p} \in \operatorname{supp}_{A}(M)$, and height $(A / \mathfrak{p})+$ $\operatorname{dim}(A / \mathfrak{p})=\operatorname{dim}(A)$.

Corollary 4.3. Let $(R, \mathfrak{m})$ be a universally catenary integral domain and let $E$ be a balanced big Cohen-Macaulay $R$-module. For any set $\mathbf{X}$ of indeterminates and $B=R(\mathbf{X})=R[\mathbf{X}]_{\mathfrak{m}[\mathbf{X}]}, B \otimes_{R} E$ is a balanced big Cohen-Macaulay B-module.

Theorem 4.4. Let $(R, \mathfrak{m})$ be a universally catenary integral domain containing a field. If $R$ is not Cohen-Macaulay, then $e_{1}(J)<0$ for any parameter ideal $J$.

Proof. Let $E$ be a balanced big Cohen-Macaulay $R$-module $([1,8.4 .2])$ and consider the exact sequence $0 \rightarrow R \rightarrow E \rightarrow C \rightarrow 0$. Let $\mathbf{X}$ be a set of indeterminates of larger cardinality than $\operatorname{Ass}(C)$, and let $R(\mathbf{X})=R[\mathbf{X}]_{\mathfrak{m} R[\mathbf{X}]}$. By applying Theorem 4.1 to the exact sequence $0 \rightarrow R(\mathbf{X}) \rightarrow E \otimes R(\mathbf{X}) \rightarrow C \otimes R(\mathbf{X}) \rightarrow 0$, the assertion is proved.

\section{Filtered modules}

The same relationship discussed above between the signature of $e_{1}(J)$ and the Cohen-Macaulayness of $R$ holds true when modules are examined. Recall that if a Noetherian local ring $R$ is embedded into either a maximal Cohen-Macaulay module ([12, Theorem 3.1]) or a balanced big Cohen-Macaulay module ([12, proof of Theorem $3.2]$ ), then whenever $R$ is not Cohen-Macaulay, we have $e_{1}(J)<0$ for any parameter ideal $J$. Now we use the same arguments as in [12, Theorems 3.1, 3.2] in order to extend the validity of Theorem 3.3 in the following manner.

Theorem 5.1. Let $(R, \mathfrak{m})$ be a Noetherian local ring of dimension $d \geq 1$ and let $M$ be a finitely generated module embedded in a maximal Cohen-Macaulay module E. Then $M$ is Cohen-Macaulay if and only if $e_{1}(J, M) \geq 0$ for any ideal $J$ generated by a system of parameters of $M$.

A variation that uses Theorem 4.4 is the following.

Theorem 5.2. Let $(R, \mathfrak{m})$ be a universally catenary integral domain containing a field and let $M$ be a finitely generated torsion-free $R$-module. If $M$ is not Cohen-Macaulay, then $e_{1}(J, M)<0$ for any ideal $J$ generated by a system of parameters of $M$.

Proof. By assumption $M$ is a submodule of a finitely generated free $R$-module, which can be embedded into a finite direct sum of balanced big Cohen-Macaulay modules. The argument of [12, Theorem 3.2] applies again.

Let now $R=k\left[x_{1}, \ldots, x_{d}\right]$ be a ring of polynomials over the field $k$, and let $M$ be a finitely generated graded $R$-module. Suppose $\operatorname{dim} M=d$. For $J=\left(x_{1}, \ldots, x_{d}\right)$ we can apply Theorem 5.1 to $M$ in a manner that uses the Hilbert-Samuel function information of the native grading of $M$. 
Theorem 5.3. Let $R=k[\mathbf{x}]=k\left[x_{1}, \ldots, x_{d}\right], d \geq 2$, be a ring of polynomials over the field $k$, and let $M$ be a finitely generated graded $R$-module generated in degree 0 . If $M$ is torsion-free, then $M$ is a free R-module if and only if $e_{1}(M)=0$.

Proof. Since $M$ is generated in degree $0, M \simeq \operatorname{gr}_{\mathbf{x}}(M)$. By assumption, $M$ can be embedded in a free $R$-module $E$ (not necessarily by a homogeneous homomorphism). Now we apply Theorem 5.1.

If $M$ is generated in degree $a>0$, we have the equality

$$
\lambda\left(M /(\mathbf{x})^{n+1} M\right)=\sum_{k=0}^{n} \lambda\left(M_{a+k}\right),
$$

so the Hilbert coefficients satisfy

$$
\begin{aligned}
& e_{0}\left(\operatorname{gr}_{\mathbf{x}}(M)\right)=e_{0}(M[a])=e_{0}(M), \\
& e_{1}\left(\operatorname{gr}_{\mathbf{x}}(M)\right)=e_{1}(M[a])=e_{1}(M)-a e_{0}(M) .
\end{aligned}
$$

Corollary 5.4. Let $R=k[\mathbf{x}]=k\left[x_{1}, \ldots, x_{d}\right], d \geq 2$, be a ring of polynomials over the field $k$, and let $M$ be a finitely generated graded $R$-module generated in degree $a \geq 0$. If $M$ is torsion-free, then $e_{1}(M) \leq a e_{0}(M)$, with equality if and only if $M$ is a free $R$-module.

\section{References}

[1] W. Bruns and J. Herzog, Cohen-Macaulay Rings, Cambridge University Press, 1993.

[2] R. Gilmer and W. Heinzer, The Noetherian property for quotient rings of infinite polynomial rings, Proc. Amer. Math. Soc. 76 (1979), 1-7.

[3] S. Goto and K. Nishida, Hilbert coefficients and Buchsbaumness of associated graded rings, J. Pure \& Applied Algebra 181 (2003), 61-74.

[4] S. Huckaba and T. Marley, Hilbert coefficients and the depths of associated graded rings, J. London Math. Soc. 56 (1997), 64-76.

[5] C. Huneke and B. Ulrich, General hyperplane sections of algebraic varieties, J. Algebraic Geom. 2 (1993), 487-505.

[6] M. Nagata, Local Rings, Interscience, New York, 1962.

[7] D. G. Northcott, A note on the coefficients of the abstract Hilbert function, J. London Math. Soc. 35 (1960), 209-214.

[8] C. Polini, B. Ulrich and W. V. Vasconcelos, Normalization of ideals and Briançon-Skoda numbers, Math. Research Letters, 12 (2005), 827-842.

[9] M. E. Rossi and G. Valla, Hilbert Function of Filtered Modules, arXiv:math.AC/07102346.

[10] M. E. Rossi and G. Valla, On the Chern number of a filtration, Rendiconti Seminario Matematico Padova, to appear.

[11] W. V. Vasconcelos, Reflexive modules over Gorenstein rings, Proc. Amer. Math. Soc. 19 (1968), 1349-1355.

[12] W. V. Vasconcelos, The Chern coefficients of local rings, Michigan Math. J. 57 (2008), 725-744.

[13] S. Zarzuela, Balanced big Cohen-Macaulay modules and flat extensions of rings, Math. Proc. Camb. Phil. Soc. 102 (1987), 203-209. 
Department of Mathematics, New York City College of Technology-Cuny, 300 Jay Street, Brooklyn, NY 11201, U.S.A.

E-mail address: lghezzi@citytech.cuny.edu

Department of Mathematics, Southern Connecticut State University, 501 Crescent Street, New Haven, CT 06515-1533, U.S.A.

E-mail address: hongj2@southernct.edu

Department of Mathematics, Rutgers University, 110 Frelinghuysen Rd, Piscataway, NJ 08854-8019, U.S.A.

E-mail address: vasconce@math.rutgers.edu 